\title{
gु \\ Electrostatic tuning of Kondo effect in a rare-earth-doped wide-band-gap oxide
}

\author{
Yongfeng Li, ${ }^{1,2}$ Rui Deng, ${ }^{1}$ Weinan Lin, ${ }^{1}$ Yufeng Tian, ${ }^{1}$ Haiyang Peng, ${ }^{1}$ Jiabao Yi,${ }^{3}$ Bin Yao, ${ }^{4}$ and Tom Wu ${ }^{1,5}, *$ \\ ${ }^{1}$ Division of Physics and Applied Physics, School of Physical and Mathematical Sciences, Nanyang Technological University, \\ 21 Nanyang Link, Singapore 637371, Singapore \\ ${ }^{2}$ Key Laboratory of Physics and Technology for Advanced Batteries (Ministry of Education), College of Physics, Jilin University, \\ Changchun 130012, People's Republic of China \\ ${ }^{3}$ School of Materials Science \& Engineering, The University of New South Wales, Sydney NSW 2052, Australia \\ ${ }^{4}$ State Key Lab of Superhard Material, College of Physics, Jilin University, Changchun 130012, China \\ ${ }^{5}$ Physical Sciences and Engineering Division, King Abdullah University of Science and Technology, Thuwal 23955-6900, Saudi Arabia
}

(Received 29 December 2012; published 29 April 2013)

\begin{abstract}
As a long-lived theme in solid-state physics, the Kondo effect reflects the many-body physics involving the short-range Coulomb interactions between itinerant electrons and localized spins in metallic materials. Here we show that the Kondo effect is present in $\mathrm{ZnO}$, a prototypical wide-band-gap oxide, doped with a rare-earth element (Gd). The localized $4 f$ electrons of Gd ions do not produce remanent magnetism, but interact strongly with the host electrons, giving rise to a saturating resistance upturn and negative magnetoresistance at low temperatures. Furthermore, the Kondo temperature and resistance can be electrostatically modulated using electric-double-layer gating with liquid ionic electrolyte. Our experiments provide the experimental evidence of tunable Kondo effect in $\mathrm{ZnO}$, underscoring the magnetic interactions between localized and itinerant electrons and the emergent transport behaviors in such doped wide-band-gap oxides.
\end{abstract}

DOI: 10.1103/PhysRevB.87.155151

PACS number(s): 72.15.Qm, 75.50.Pp, 75.30.Hx, 75.47.-m

\section{INTRODUCTION}

In the Kondo effect, the antiferromagnetic coupling between itinerant electrons and localized spins results in the screening of the impurity spins, manifesting in the anomalous resistance upturn at low temperatures. ${ }^{1,2}$ During the past a few decades, the research efforts have been providing insights on the intriguing physics of strongly correlated electron systems such as heavy-fermion compounds and high- $T_{c}$ superconductors. Although the Kondo effect was first discovered in normal metals containing minute amounts of magnetic impurities, ${ }^{3}$ recent experiments suggest its existence in several oxide- and carbon-based materials, including doped $\mathrm{TiO}_{2}$ thin films ${ }^{4,5} \mathrm{SrTiO}_{3},{ }^{6}$ and graphene. ${ }^{7}$ These discoveries challenge the theoretical models which were originally developed to describe classic Kondo systems like $\mathrm{Fe}$ impurities in $\mathrm{Au}$ thin films. To develop a realistic model, multiple effects like orbital structure, Hund's coupling, and electron hybridization must be considered collectively. In terms of systems which manifest Kondo physics, it is noteworthy that so far there has been no report on the Kondo effect in zinc oxide $(\mathrm{ZnO})$, a prototypical wide-band-gap oxide with prominent optical and electrical properties. Furthermore, because the spin-flip scattering of conduction electrons by dilute magnetic impurities is strongly dependent on the carrier concentration in the conduction band, the Kondo effect can potentially be tuned electrostatically in a field-effect transistor (FET). An important advantage of such research is that in a FET the charge-carrier density can be modulated without introducing chemical or structural disorder. ${ }^{8}$ However, for the conventional metaloxide-semiconductor field-effect transistors (MOSFET), the breakdown of gate dielectrics limits the maximum sheet carrier density that can be induced (usually $<10^{13} \mathrm{~cm}^{-2}$ ), ${ }^{9}$ which calls for novel materials and device architectures.

Here, we demonstrate the presence of the Kondo effect in a rare-earth-doped wide-band-gap oxide, i.e., Gd-doped
$\mathrm{ZnO}$, which is metallic and paramagnetic. Gd doping allegedly induces colossal magnetic moments in $\mathrm{GaN}$, a well-studied wide-band-gap semiconductor. ${ }^{10} \mathrm{~A}$ recent theoretical study predicts a Kondo-like resonance, namely, a narrow resonant $f$ band near the Fermi level in Eu-doped $\mathrm{ZnO} .{ }^{11}$ Furthermore, we show that the Kondo behavior in Gd-doped $\mathrm{ZnO}$ can be electrostatically tuned in an electric-double-layer transistor (EDLT) using an ionic gel electrolyte. Although the $4 f$ electrons of rare-earth elements are localized, our results suggest a strong correlation between their high orbital moments with the itinerant carriers, and the resulting Kondo effect can be tailored by the high-density electron accumulation.

\section{EXPERIMENT}

We synthesized undoped, 1.0, 1.8, and 3.4 at. \% Gddoped $\mathrm{ZnO}$ films with thickness of about $80 \mathrm{~nm}$ on $a$-plane single-crystal sapphire substrates using pulsed laser deposition (PLD). The synthesis procedures are similar to our previous reports on doped $\mathrm{ZnO}$ films. ${ }^{12-20}$ During the growth process, the substrate temperature was fixed at $450{ }^{\circ} \mathrm{C}$, and the laser repetition rate was $2 \mathrm{~Hz}$. The oxygen pressure was maintained at $1 \times 10^{-4}$ and $4 \times 10^{-4} \mathrm{~Pa}$ for the undoped and the Gd-doped $\mathrm{ZnO}$ films, respectively.

We first checked the structural properties of the Gd-doped $\mathrm{ZnO}$ films using $\mathrm{x}$-ray diffraction (XRD). Figure 1(a) shows the XRD patterns of the pure and Gd-doped $\mathrm{ZnO}$ thin films. All films present the wurtzite structure with a (002) preferential orientation and no impurity phase is observed. The enlarged view of the $\mathrm{ZnO}$ (002) diffraction peaks is shown in Fig. 1(b). A shift of the diffraction peaks to low angles indicates that the $c$-axis lattice constant increases with the Gd concentration. Figure 1(c) shows the Gd-concentration dependence of the out-of-plane strain obtained from both the XRD data and the first-principles calculation. Clearly, the tensile strain in the $c$ 

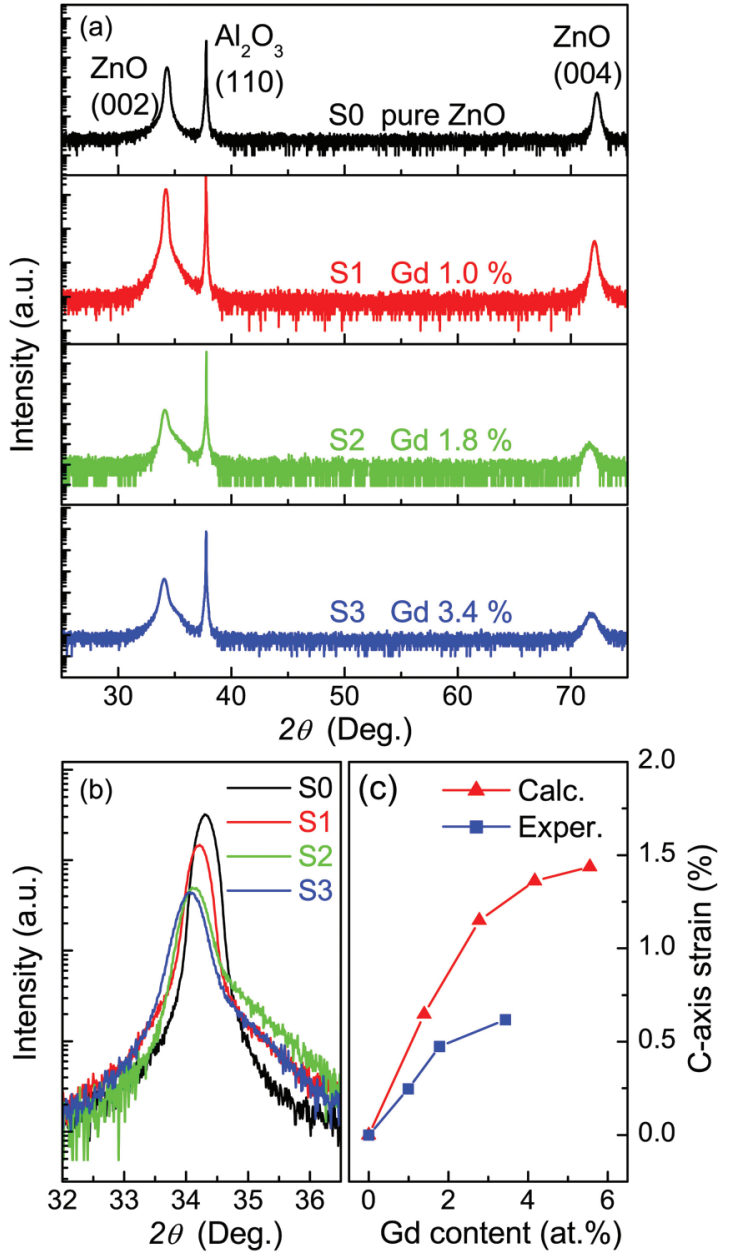

FIG. 1. (Color online) (a) XRD patterns of the pure and the Gddoped $\mathrm{ZnO}$ thin films, indicating that the Gd-doped $\mathrm{ZnO}$ films have the single wurtzite structure with a (002) preferential orientation, and no impurity phase was observed for all samples. (b) Enlarged view of the $\mathrm{ZnO}(002)$ diffraction peaks. A shift of the diffraction peaks to low diffraction angles indicates that the $c$-axis lattice constant increases with the Gd concentration. (c) Gd-concentration dependence of the out-of-plane strain obtained from both the XRD data and the firstprinciples calculation. The tensile strain in the $c$ axis increases with the $\mathrm{Gd}$ concentration as a result of the larger atom radius of $\mathrm{Gd}$.

axis increases with the Gd concentration, which results from the larger atom radius of the Gd ions compared to that of the $\mathrm{Zn}$ ions. In addition, we characterized the Gd-doped $\mathrm{ZnO}$ films using x-ray photoelectron spectroscopy, optical absorption, and photoluminescence techniques. ${ }^{21}$

In our field effect experiments, we used the undoped and the 1.8 at. \% Gd-doped $\mathrm{ZnO}$ as channels in EDLT devices. Recently, as a result of its capability to generate enormous electrical fields, EDLT has been extensively used to discover and modulate novel physical properties. ${ }^{22-28}$ To form the channel, we used a shadow mask with the Hall-bar pattern during the film deposition, and the configuration of the planar device is shown in Fig. 2(a). Indium metal served as the ohmic contact to the patterned films. To avoid excessive leakage current, we used resin to isolate the electrodes and wirings from the electrolyte [electrolyte, N, N-diethyl-N-methyl-N-(2methoxyethyl)ammonium bis(trifluoromethylsulfonyl)imide
(DEME-TFSI)]. We also made devices with other Gd doping levels, but found that the tuning is much weaker when the Gd levels and the resulting conductivity are too high. A drop of ionic liquid electrolyte, DEME-TFSI, covers the channel and the gate electrode. As shown in the schematic in Fig. 2(b), the field-induced drift and distribution of cations, $\left(\mathrm{CH}_{3} \mathrm{CH}_{2}\right)_{2}\left(\mathrm{CH}_{2} \mathrm{CH}_{2} \mathrm{OCH}_{3}\right) \mathrm{CH}_{3} \mathrm{~N}^{+}$(DEME), and anions, $\left(\mathrm{CF}_{3} \mathrm{SO}_{2}\right)_{2} \mathrm{~N}^{-}$(TFSI), accomplish the electrostatic gating.

The transport measurements at various gate voltages $\left(V_{\mathrm{G}}\right)$ were conducted in a physical properties measurement system (PPMS, Quantum Design). A Keithley 4200 semiconductor characterization system was used to collect the current-voltage and capacitance data. Because the ionic liquid is frozen at $\sim 195 \mathrm{~K}$, we first applied $V_{\mathrm{G}}$ at $230 \mathrm{~K}$ for $30 \mathrm{~min}$ to accumulate the carriers at the channel and then collected the resistance vs temperature $(R-T)$ data during cooling down. Before switching to a different $V_{\mathrm{G}}$, the device was warmed up to $230 \mathrm{~K}$.

In the superconducting quantum interference device (SQUID) magnetometer measurements, the diamagnetic backgrounds of the sapphire substrates were carefully calibrated and subtracted from the raw data. Polarized $\mathrm{x}$-ray-absorption spectrum (XAS) and x-ray magnetic circular dichroism (XMCD) measurements at the Gd $M_{4,5}$ edge (transitions from $3 d^{10} 4 f^{7}$ to $3 d^{9} 4 f^{8}$ ) were carried out under a magnetic field of $4 \mathrm{~T}$ at the WERA beamline in ANKA, Kalsruhe Institute Technology University (KIT), Germany.

In the first-principles density functional (DFT) calculations, we used the VASP $\operatorname{code}^{29,30}$ with the projector augmented wave (PAW) potentials for the electronic interaction and the generalized gradient approximation (GGA) for the electron exchange and correlation. ${ }^{31,32}$ The cutoff energy for the plane-wave basis set is $400 \mathrm{eV}$. We constructed a 72-atom $3 \times 3 \times 2$ supercell with the wurtzite structure. To simulate the Gd-doped $\mathrm{ZnO}$, two nearest-neighbor $\mathrm{Zn}$ atoms are substituted by $\mathrm{Gd}$ atoms in the supercell $\left(\mathrm{Gd}_{2} \mathrm{Zn}_{34} \mathrm{O}_{36}\right)$, corresponding to a $\mathrm{Gd}$ concentration of 2.8 at. \%. For the Brillouin-zone integration, a $3 \times 3 \times 3$ Monkhorst-Pack $k$-point mesh was used; a more refined $(8 \times 8 \times 8) k$-point mesh was used for the density-of-states (DOS) calculations. In the calculations, all the atoms are allowed to relax until the Hellmann-Feynman forces acting on them become less than $0.01 \mathrm{eV} / \AA$.

\section{RESULTS AND DISCUSSION}

Figures 2(c) and 2(d) show the temperature-dependent resistance data measured in undoped and $\mathrm{Gd}$-doped $\mathrm{ZnO}$ EDLTs. Due to the electron accumulation, the resistance of the $n$-type channel decreases as $V_{\mathrm{G}}$ crosses over from negative to positive. We measured 12 devices and observed reproducible behaviors. Furthermore, the zero-gate $R-T$ curve measured at the end of the sequence almost completely overlaps with the pristine one, suggesting that the resistance modulation originates from electrostatic charge accumulation rather than electrochemical reaction. ${ }^{33}$ For the undoped $\mathrm{ZnO}$ EDLT, the $R-T$ curve illustrates a semiconductor behavior and the resistance can be tuned about two orders of magnitude as $V_{\mathrm{G}}$ varies from 0 to $2 \mathrm{~V}$ [Fig. 2(c)]. On the other hand, Gd-doped $\mathrm{ZnO}$ shows a metallic behavior with its resistance being four orders smaller than that of undoped one [Fig. 2(d)], and an 
(a)

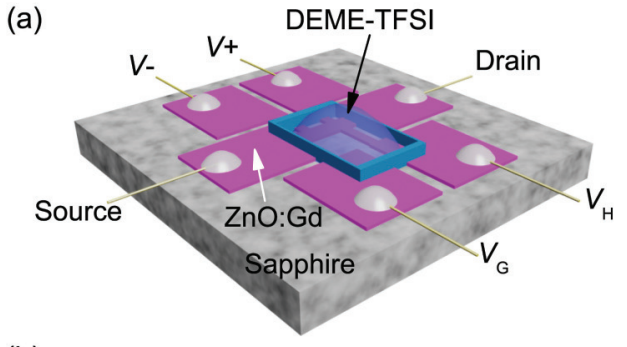

(b)

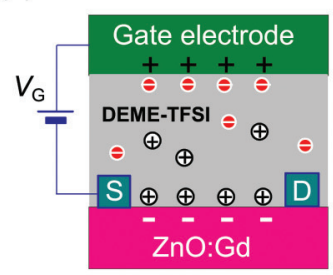

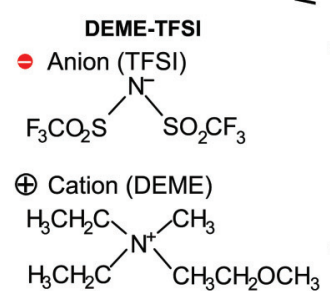

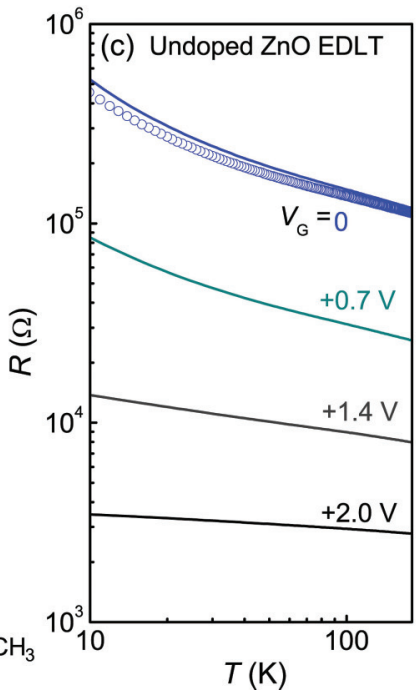

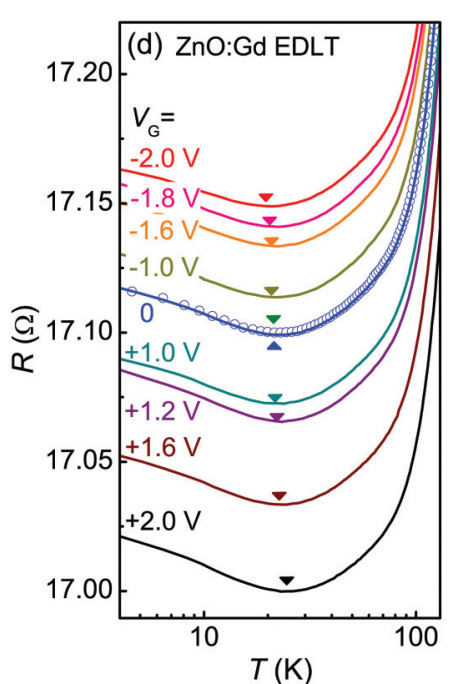

FIG. 2. (Color online) Electric double-layer transistor (EDLT) with the undoped and the Gd-doped ZnO thin films as channels. (a) Schematic diagram of the EDLT with a drop of ionic liquid electrolyte, DEME-TFSI, covering the channel and the gate electrodes. As the channel, ZnO thin films were grown on $a$-plane sapphire substrates and patterned into Hall bars. (b) Schematic illustrating the charge accumulation at the interface between the liquid electrolyte and the oxide channel. The field-induced drift and distribution of cations, $\left(\mathrm{CH}_{3} \mathrm{CH}_{2}\right)_{2}\left(\mathrm{CH}_{2} \mathrm{CH}_{2} \mathrm{OCH}_{3}\right) \mathrm{CH}_{3} \mathrm{~N}^{+}$ (DEME), and anions, $\left(\mathrm{CF}_{3} \mathrm{SO}_{2}\right)_{2} \mathrm{~N}^{-}$(TFSI), accomplish the electrostatic gating. (c),(d) Temperature dependence of channel resistances measured under various gate voltages $\left(V_{\mathrm{G}}\right)$ for pure $\mathrm{ZnO}$ and 1.8 at. \% Gd-doped $\mathrm{ZnO}$, respectively. In the measurement sequence, increasing positive $V_{\mathrm{G}}$ was applied, and after the maximum $V_{\mathrm{G}}$ the $R-T$ curve at $V_{\mathrm{G}}=0$ (circles) was remeasured; the almost complete overlap of the second zero-gate curve with the pristine one suggests negligible electrochemical reaction at the channel/electrolyte interface.

upturn of resistance was observed at low temperatures. ${ }^{34} \mathrm{Gd}$ ions are known to introduce magnetic moments in the host, so we hypothesize that the magnetism plays a role in transport. Recently, there have been intense interest on doping $\mathrm{ZnO}$ with transition-metal and rare-earth elements to activate the magnetic degrees of freedom, ${ }^{35,36}$ but open questions remain regarding the interaction between itinerant carriers and local magnetic moments.

Although both the Kondo effect and weak localization effect can lead to resistance minimum and upturn at low temperature, weak localization effect exhibits resistance divergence, ${ }^{37}$ which differs from the characteristics of the Kondo effect. ${ }^{38}$ In Fig. 3(a), the temperature-dependent resistance data at $V_{\mathrm{G}}=0$ show saturating upturn at $T<5 \mathrm{~K}$, indicating that Kondo scattering dominates the transport in Gd-doped $\mathrm{ZnO}$. We can describe the temperature-dependent resistance as

$$
R(T)=R_{0}+q T^{2}+p T^{5}+R_{K}(0 \mathrm{~K})\left[T_{K}^{\prime 2} /\left(T^{2}+T_{K}^{\prime 2}\right)\right]^{s},
$$

where $R_{0}$ is the residual resistance, and the second and the third terms represent the contributions of electron-electron and electron-phonon interactions, respectively. In the last term, $T_{K}^{\prime}=T_{K}\left(V_{G}\right) /\left(2^{1 / s}-1\right)^{1 / 2}$, where $T_{K}$ is the Kondo temperature and $s=0.225$ according to the result obtained from the numerical renormalization-group (NRG) theory. ${ }^{39,40}$ The fitting result using Eq. (1) is shown in Fig. 3(a) and the obtained fitting parameters are $R_{0}=17.07 \Omega, q=1.01 \times$ $10^{-5} \Omega / \mathrm{K}^{2}, p=1.04 \times 10^{-11} \Omega / \mathrm{K}^{5}, R_{\mathrm{K}}(0 \mathrm{~K})=0.055 \Omega$, $T_{\mathrm{K}}=22.01 \mathrm{~K}$.

As shown in Fig. 2(d), the gate voltage has a clear modulation effect on the channel transport. Since the empirical temperature-dependent Kondo resistance, $R_{K}(T)=R_{K}(0 \mathrm{~K})\left[T_{K}^{\prime 2} /\left(T^{2}+T_{K}^{\prime 2}\right)\right]$ can describe the universal behavior, ${ }^{39}$ we scaled the $R(T)$ curves obtained with various $V_{\mathrm{G}}$ values to a normalized form. Figure 3(b) shows the plot of scaled resistance $R_{\mathrm{K}}(T) / R_{\mathrm{K}}(0)$, namely, $\left[R(T)-R_{0}-\right.$ $\left.q T^{2}-p T^{5}\right] / R_{\mathrm{K}}(0)$, versus $T / T_{\mathrm{K}}$. The experimental data fit well to the universal Kondo behavior at $T<T_{\mathrm{K}}$, and at high temperatures the deviation with respect to the NRG result can be attributed to the phonon scattering.

It is well established that the Kondo resistance upturn can be suppressed by a magnetic field [Fig. 3(c)], which was reported previously for dilute magnetic Kondo alloys. ${ }^{41}$ The strength of resistance upturn, as represented by $R(T)-R(10 \mathrm{~K})$ in the inset of Fig. 3(c), is significantly reduced as the applied magnetic field increases. The resistance minimum also shifts to lower temperatures. A negative magnetoresistance was observed at temperatures below the Kondo temperature [Fig. 3(d)], which can be attributed to the splitting of the Kondo peak in the spectral density by the applied magnetic field and the subsequent suppression of Kondo effect in transport. ${ }^{42}$ Since the Kondo temperature for the Gd-doped $\mathrm{ZnO}$ film is $22 \mathrm{~K}$, its energy scale $k_{\mathrm{B}} T_{\mathrm{K}}$ corresponds to a magnetic field of $\sim 32 \mathrm{~T}$. This field is much larger than the magnetic fields applied in our experiments; consequently, we did not observe the complete collapse of the Kondo effect.

We now address the origin of the localized magnetic moments in $\mathrm{Gd}$-doped $\mathrm{ZnO}$ which are responsible for the scattering of conduction electrons in the Kondo effect. Figure 4(a) shows the temperature-dependent magnetization ( $M$ - $T$ curve) of the 1.8 at. \% Gd-doped $\mathrm{ZnO}$ film measured under an applied magnetic field of 1000 Oe in both the zero-field-cooled (ZFC) and the field-cooled (FC) modes. The overlap of the ZFC and 

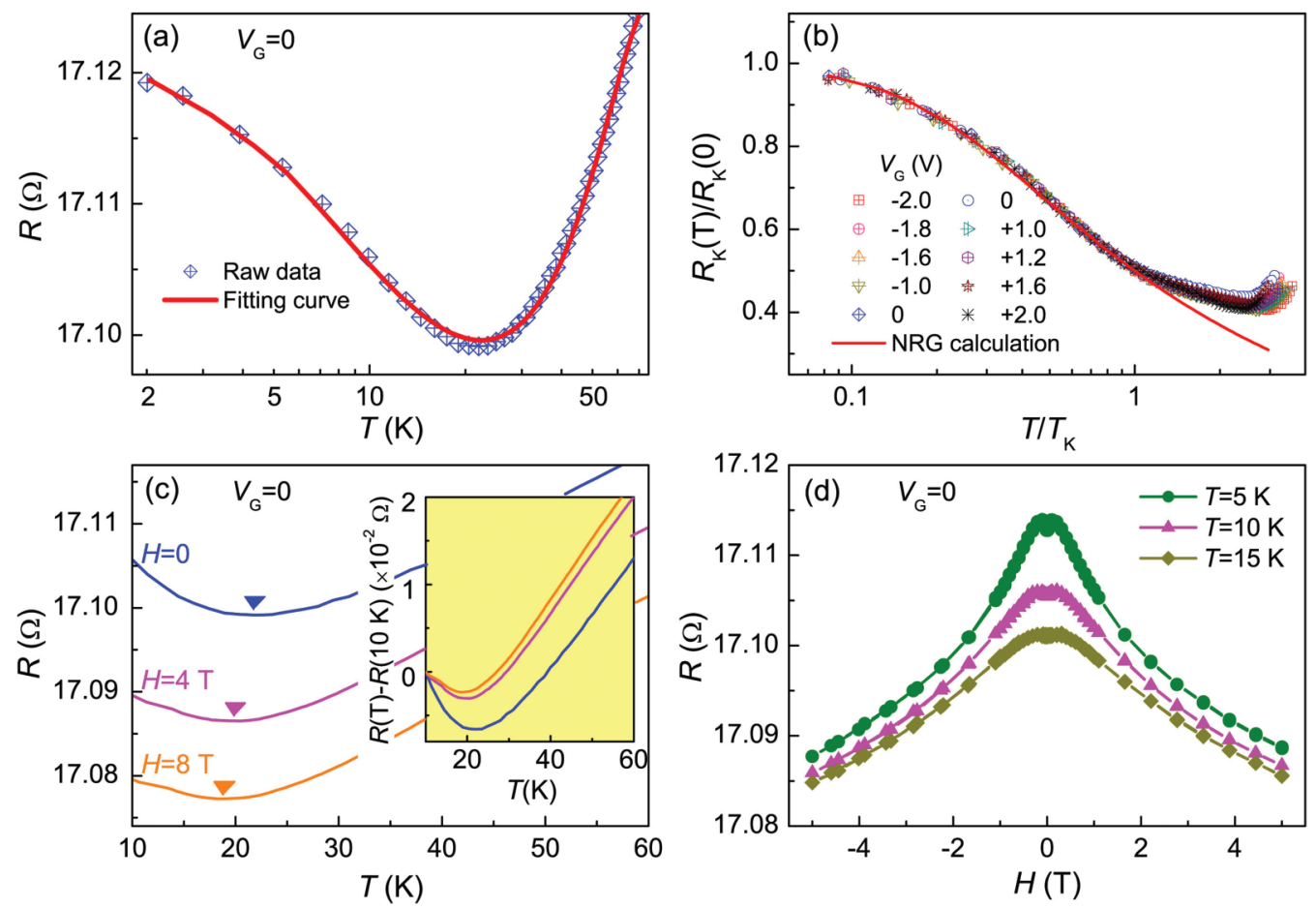

FIG. 3. (Color online) Transport and Kondo effect in the Gd-doped $\mathrm{ZnO}$ channel. (a) Temperature dependence of channel resistance at $V_{\mathrm{G}}=0$. Fitting to Eq. (1) (solid curve) gives parameters $R_{0}=17.07 \Omega, q=1.01 \times 10^{-5} \Omega / \mathrm{K}^{2}, p=1.04 \times 10^{-11} \Omega / \mathrm{K}^{5}, R_{\mathrm{K}}(0 \mathrm{~K})=0.055 \Omega$, and $T_{\mathrm{K}}=22.01 \mathrm{~K}$. (b) Universal Kondo behavior of the normalized resistivity $\left[R(T)-R_{0}-q T^{2}-p T^{5}\right] / R(0)$ versus $T / T_{\mathrm{K}}$ measured at various $V_{\mathrm{G}}$. The red line illustrates the universal Kondo curve from the numerical renormalization-group calculation. (c) Temperature dependence of the channel resistance at $H=0,4$, and $8 \mathrm{~T}$. The magnetic field suppresses the Kondo resistance upturn at low temperatures. The inset shows the temperature-dependent data of the resistance modulation, i.e., $R(T)-R(10 \mathrm{~K})$. (d) Negative magnetoresistance observed at $T=5,10$, and $15 \mathrm{~K}$.

FC data in the whole temperature range and the good fit to the Cure-Weiss law suggest a paramagnetic nature of Gd-doped $\mathrm{ZnO}$. The magnetization versus magnetic field $(M-H)$ curve measured at $5 \mathrm{~K}$ [inset of Fig. 4(a)] shows no hysteresis, in line with the paramagnetism. Furthermore, XMCD data measured at $300 \mathrm{~K}$ [Fig. 4(b)] confirms that there is no exchange interaction between the Gd ions, which is consistent with the previous reports. ${ }^{43}$ The absence of ferromagnetism in $\mathrm{Gd}$-doped $\mathrm{ZnO}$ is in contrast with the previous report of a strong ferromagnetic order in another rare-earth-doped oxide, i.e., $\mathrm{Nd}$-doped $\mathrm{ZnO}$ nanowires. ${ }^{44}$

To understand the band structure of Gd-doped $\mathrm{ZnO}$, we carried out first-principles DFT calculations. Figure 4(c) shows the calculated total and partial spin-resolved density of states of the $\mathrm{Gd}_{2} \mathrm{Zn}_{34} \mathrm{O}_{36}$ system. There is a significant spin splitting in the conduction band, which is contributed by the Gd- $4 f$ states. Furthermore, we obtained a magnetic moment of $6.5 \mu_{\mathrm{B}}$ for each $\mathrm{Gd}$ ion, providing evidence for the high magnetic moments in the Kondo scattering of delocalized electrons. The calculated energy difference between the antiferromagnetic and the ferromagnetic states, or $\Delta E_{\mathrm{AFM}-\mathrm{FM}}$, is only $0.6 \mathrm{meV}$, indicating that a stable ferromagnetic order cannot established, which is consistent with the experimentally observed paramagnetism. In addition, the Fermi level lies in the conduction band and close to the Gd- $f$ states, suggesting a strong interaction between the conducting electrons and the magnetic moments of Gd- $f$ orbital. ${ }^{45}$ It is also noteworthy that a resistance upturn at low temperatures was also observed in $\mathrm{ZnO}$ thin films doped with nonmagnetic Ga ions. ${ }^{46}$ But in that case, a linear variation of conductivity with $\sqrt{T}$ below the metal-insulator transition suggests that the degenerate electrons are in the weak localization regime. This further underscores the dispensable role of rare-earth Gd ions with $f$ electrons in producing the Kondo effect in the $\mathrm{ZnO}$ host.

Next we address the effect of electrostatic gating on the Kondo effect in the EDLT. As shown in Fig. 5(a), the resistance minimum $\left(T_{\min }\right)$ gradually shifts to high temperatures as the gate voltage $V_{\mathrm{G}}$ increases, indicating an enhanced Kondo scattering at higher carrier densities. The magnitude of the resistance upturn, described by $R(4 \mathrm{~K})-R_{\min }$, also becomes substantially larger at higher $V_{\mathrm{G}}$. The shift of the Kondo temperature and the magnitude of resistance upturn as a function of $V_{\mathrm{G}}$ are summarized in Fig. 5(b). The Kondo temperature $T_{\mathrm{K}}$, below which the impurity spins become screened and a Fermi liquid develops, has the following relationship with the density of states at the Fermi level $D\left(E_{\mathrm{F}}\right)$ :

$$
k_{B} T_{K} \sim e^{-1 /\left[J D\left(E_{F}\right)\right]},
$$

where $k_{\mathrm{B}}$ is the Boltzmann's constant and $J>0$ is the antiferromagnetic coupling constant. ${ }^{2}$ Accordingly, the gateinduced electron accumulation in the $\mathrm{Gd}$-doped $\mathrm{ZnO}$ channel enhances $D\left(E_{\mathrm{F}}\right)$, and as a result $T_{\mathrm{K}}$ increases.

It is noteworthy that the Kondo resistance of $R(4 \mathrm{~K})-R_{\min }$ show a $V_{\mathrm{G}}$-dependent trend opposite to the conventional metal-insulator transition in a disordered system where a 

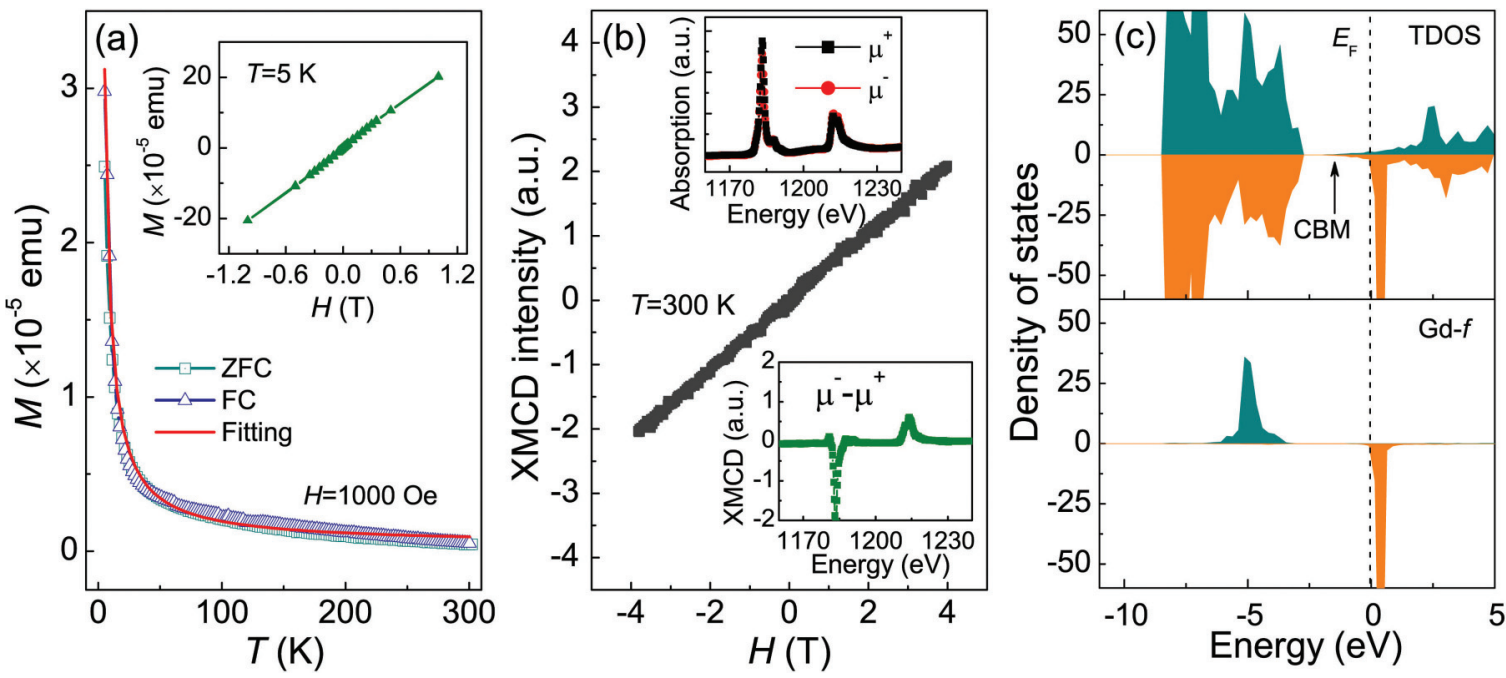

FIG. 4. (Color online) Magnetic properties of Gd-doped ZnO. (a) Temperature dependence of magnetization of the 1.8 at. \% Gd-doped $\mathrm{ZnO}$ thin film measured by SQUID under both the zero-field-cooled (ZFC) and field-cooled (FC) conditions. The solid curve shows the fitting to the Cure-Weiss law, indicating a paramagnetic behavior. The inset shows the $M-H$ curve at $5 \mathrm{~K}$. (b) XMCD data measured at $300 \mathrm{~K}$ shows no hysteresis, and it is consistent with the paramagnetic nature of the Gd-doped $\mathrm{ZnO}$. The upper-left and bottom-right insets show the polarized x-ray-absorption spectra and the XMCD signals at the $\mathrm{Gd} M_{4,5}$ edge $(H=4 \mathrm{~T})$, respectively. (c) Spin-resolved density of states calculated for the $\mathrm{Gd}_{2} \mathrm{Zn}_{34} \mathrm{O}_{36}$ system. The upper and lower panels show the total and partial density of states, respectively. The Fermi level lies in the conduction band and close to the Gd- $f$ level, indicating the energetic proximity between the conduction electrons and the local magnetic moments of Gd- $f$ orbital.

higher carrier concentration would promote the metallic state and suppress the resistance upturn. Furthermore, fitting to the three-dimensional form of electron-electron interaction (EEI) allowed us to exclude it as the origin of quantum correction
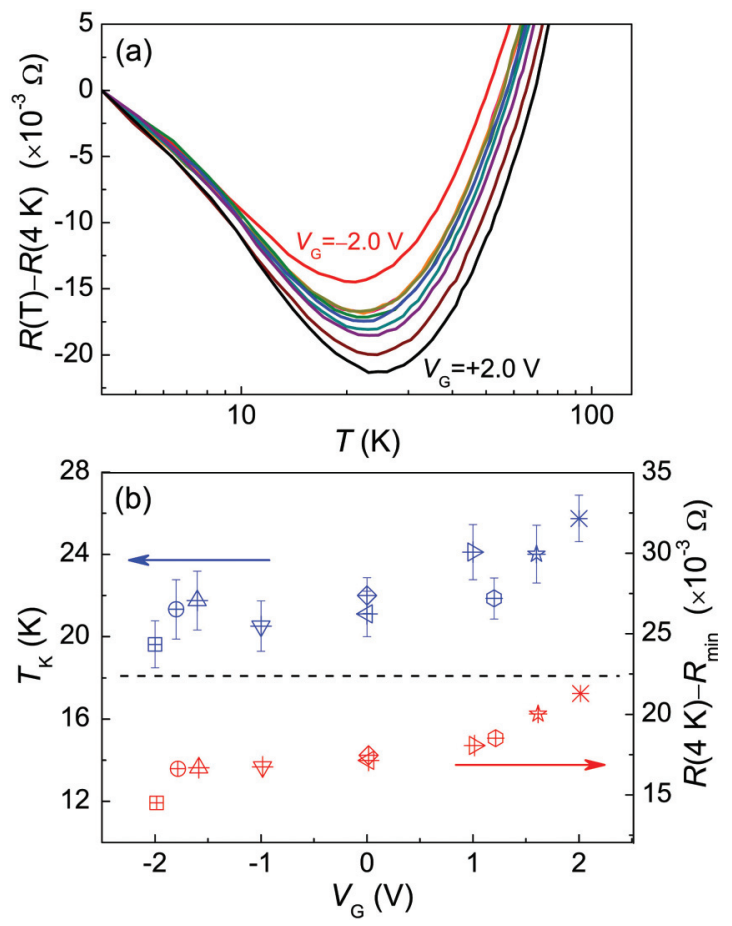

FIG. 5. (Color online) Gate-modulated Kondo effect in Gddoped $\mathrm{ZnO}$. (a) Temperature dependence of the normalized channel resistance, $R(T)-R(4 \mathrm{~K})$, measured while applying various gate voltages. (b) Gate tuning of the Kondo temperature $T_{\mathrm{K}}$ and the upturn resistance $R(4 \mathrm{~K})-R_{\min }$. to the conductivity of Gd-doped $\mathrm{ZnO}$. The contributions to the conductivity due to the EEI in the three-dimensional (3D) regime can be expressed as ${ }^{37,47}$

$$
\Delta \sigma_{e e}=-\left[0.46\left(\frac{4}{3}-\frac{3 F_{\sigma}}{2}\right)\right] \frac{e^{2}}{2 \pi^{2} \hbar}\left(\frac{1}{l}-\frac{1}{L_{T}}\right),
$$

where $F_{\sigma}$ is the correction factor, $l$ is temperature-independent cutoff length, $L_{\mathrm{T}}$ is the thermal diffusion length which can be expressed as $L_{\mathrm{T}}=\left(\hbar D / k_{B} T\right)^{1 / 2}$ with $D$ as the diffusion constant. Figure 6 shows the temperature-dependent conductivity of the EDLT with the $1.8 \%$ Gd-doped $\mathrm{ZnO}$ channel, and it is clear that Eq. (3) does not present a satisfactory fitting to the experimental data. Therefore, we can conclude

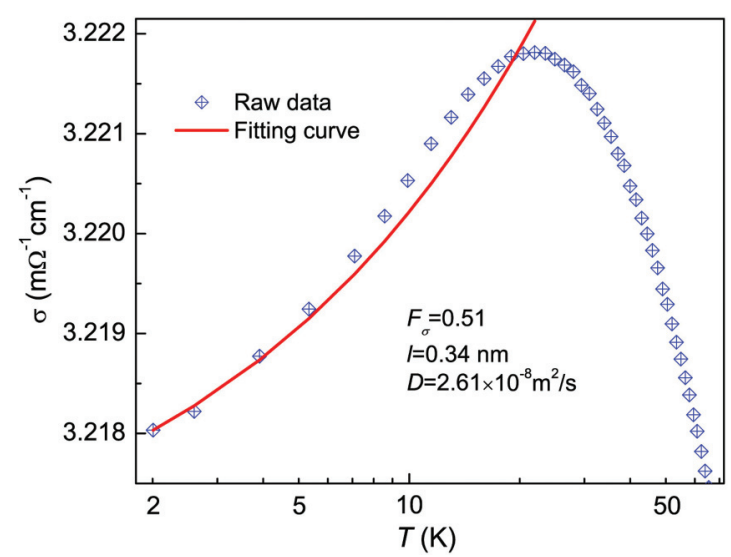

FIG. 6. (Color online) Temperature dependence of the conductivity of the $1.8 \% \mathrm{Gd}$-doped $\mathrm{ZnO}$. The red solid curve is the best fitting to Eq. (3). The fitting parameters are $F_{\sigma}=0.51, l=0.34 \mathrm{~nm}$, and $D=2.61 \times 10^{-8} \mathrm{~m}^{2} / \mathrm{s}$. It is clear that the EEI does not dominate the transport character in Gd-doped $\mathrm{ZnO}$. 

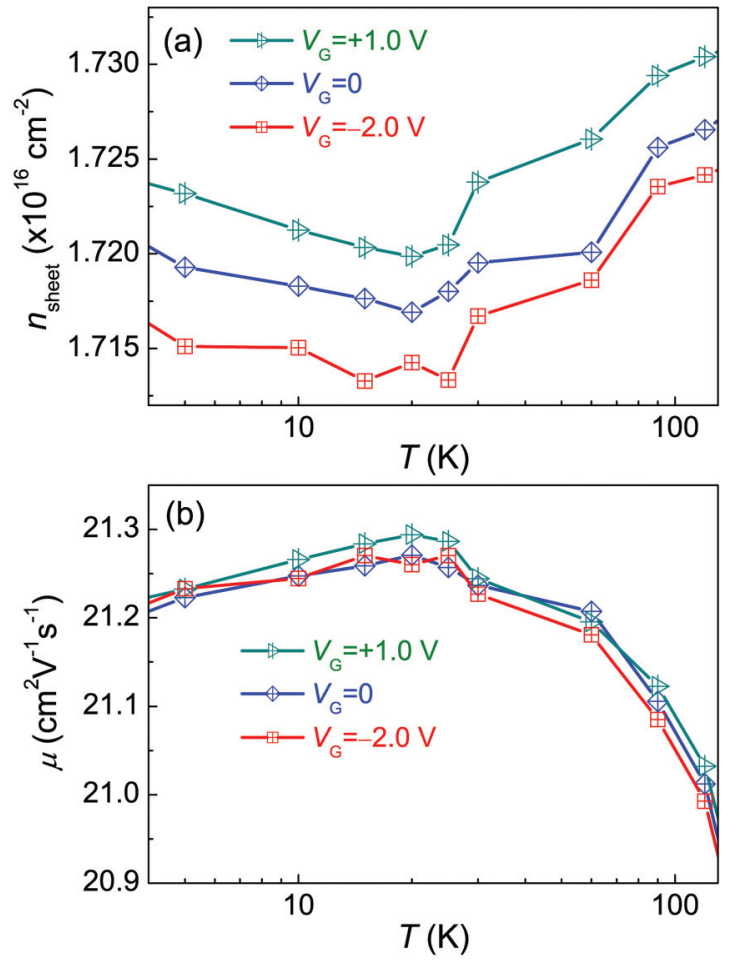

FIG. 7. (Color online) Temperature and gate dependence of (a) the sheet carrier density $n_{\text {sheet }}=-1 / R_{\mathrm{H}} e$ and (b) the mobility $\mu$, respectively.

that the Kondo effect is a more suitable scenario to describe the low-temperature transport character in Gd-doped $\mathrm{ZnO}$.

Figure 7(a) shows the temperature-dependent sheet carrier density $n_{\text {sheet }}$ measured by the Hall effect. In Gd-doped $\mathrm{ZnO}$, $n_{\text {sheet }}$ is about $1.7 \times 10^{16} \mathrm{~cm}^{-2}$. The dependence of $n_{\text {sheet }}$ on $V_{\mathrm{G}}$ verifies the carrier modulation by the gate voltages. The magnitude of modulation $\Delta n_{\text {sheet }}$ is close to $10^{14} \mathrm{~cm}^{-2}$, which is consistent with the results of capacitance measurements performed on the EDLT devices. ${ }^{48}$ On the other hand, the Hall mobility $\mu$ in Fig. 7(b) shows a much weaker dependence on $V_{\mathrm{G}}$. As the temperature goes down, the Hall mobility first increases up to $T_{\mathrm{K}}$ and then decreases, indicating that the phonon scattering and the Kondo scattering dominate the high and low temperature regimes, respectively. These observations of carrier dependence put constraint on the theoretical models in describing the Kondo physics in rare-earth-doped wideband-gap oxides.

\section{CONCLUSIONS}

Unlike the paramagnetic metals imbedded with magnetic impurities where the Kondo effect was originally discovered more than half a century ago, $\mathrm{ZnO}$ is not a conventional material for manifesting the Kondo physics. However, by doping $\mathrm{Gd}$ ions with high magnetic moments, our work unambiguously added $\mathrm{ZnO}$ as a new member to the family of Kondo materials. In a broad perspective, doping rare-earth elements with localized orbital moments and introducing indirect exchange interactions could be a reliable approach to induce the Kondo effect in wide-band-gap oxides and to explore the many-body physics with short-range Coulomb interactions. Furthermore, as we demonstrated here, the ability to tune the Kondo effect electrostatically in an EDLT without introducing any chemical disorder will open doors toward investigating the emergent physics between itinerant carriers and local magnetic moments in strongly correlated electron systems.

\section{ACKNOWLEDGMENTS}

This work was supported in part by the National Research Foundation of Singapore, Australian Research Council Discovery Program Queen Elizabeth II Fellowship Grant No. DP1105338, and the Ph.D. Programs Foundation of the Ministry of Education of China (Grant No. 20120061120011). The authors also acknowledge the assistance of XMCD measurements in ANKA, Germany. *tao.wu@kaust.edu.sa

${ }^{1}$ J. Kondo, Prog. Theor. Phys. 32, 37 (1964).

${ }^{2}$ A. C. Hewson, The Kondo Problem to Heavy Fermions (Cambridge University Press, London, 1993).

${ }^{3}$ W. J. de Haas, J. de Boer, and G. J. van dën Berg, Physica 1, 1115 (1934).

${ }^{4}$ S. Zhang, S. B. Ogale, W. Yu, X. Gao, T. Liu, S. Ghosh, G. P. Das, A. T. S. Wee, R. L. Greene, and T. Venkatesan, Adv. Mater. 21, 2282 (2009).

${ }^{5}$ R. Ramaneti, J. Lodder, and R. Jansen, Phys. Rev. B 76, 195207 (2007).

${ }^{6}$ M. Lee, J. Williams, S. Zhang, C. Frisbie, and D. GoldhaberGordon, Phys. Rev. Lett. 107, 256601 (2011).

${ }^{7}$ J.-H. Chen, L. Li, W. G. Cullen, E. D. Williams, and M. S. Fuhrer, Nat. Phys. 7, 535 (2011).

${ }^{8}$ C. H. Ahn, J. M. Triscone, and J. Mannhart, Nature (London) 424, 1015 (2003).
${ }^{9}$ D. E. Kotecki, Integr. Ferroelec. 16, 1 (1997).

${ }^{10}$ L. Liu, P. Yu, Z. Ma, and S. Mao, Phys. Rev. Lett. 100, 127203 (2008).

${ }^{11}$ A. Blanca-Romero, M. Berrondo, and J. F. Rivas-Silva, Int. J. Quantum Chem. 111, 3831 (2011).

${ }^{12}$ Y. F. Li, R. Deng, B. Yao, G. Z. Xing, D. D. Wang, and T. Wu, Appl. Phys. Lett. 97, 102506 (2010).

${ }^{13}$ Y. Tian, Y. Li, M. He, I. A. Putra, H. Peng, B. Yao, S. A. Cheong, and T. Wu, Appl. Phys. Lett. 98, 162503 (2011).

${ }^{14}$ Y.-F. Tian, Y.-F. Li, and T. Wu, Appl. Phys. Lett. 99, 222503 (2011).

${ }^{15}$ Y. F. Li, B. Yao, Y. M. Lu, B. H. Li, Y. Q. Gai, C. X. Cong, Z. Z. Zhang, D. X. Zhao, J. Y. Zhang, D. Z. Shen, and X. W. Fan, Appl. Phys. Lett. 92, 192116 (2008).

${ }^{16}$ B. Y. Zhang, B. Yao, Y. F. Li, A. M. Liu, Z. Z. Zhang, B. H. Li, G. Z. Xing, T. Wu, X. B. Qin, D. X. Zhao, C. X. Shan, and D. Z. Shen, Appl. Phys. Lett. 99, 182503 (2011). 
${ }^{17}$ B. Y. Zhang, B. Yao, Y. F. Li, Z. Z. Zhang, B. H. Li, C. X. Shan, D. X. Zhao, and D. Z. Shen, Appl. Phys. Lett. 97, 222101 (2010).

${ }^{18}$ Y. Tian, W. Lin, and T. Wu, Appl. Phys. Lett. 100, 052408 (2012).

${ }^{19}$ M. He, Y. F. Tian, D. Springer, I. A. Putra, G. Z. Xing, E. E. M. Chia, S. A. Cheong, and T. Wu, Appl. Phys. Lett. 99, 222511 (2011).

${ }^{20}$ Y. F. Li, B. Yao, R. Deng, B. H. Li, J. Y. Zhang, Y. M. Zhao, D. Y. Jiang, Z. Z. Zhang, C. X. Shan, D. Z. Shen, X. W. Fan, and Y. M. Lu, J. Phys. D: Appl. Phys. 42, 105102 (2009).

${ }^{21}$ See Supplemental Material at http://link.aps.org/supplemental/ 10.1103/PhysRevB.87.155151 for x-ray photoelectron spectroscopy, optical absorption, and photoluminescence data.

${ }^{22}$ K. Ueno, S. Nakamura, H. Shimotani, A. Ohtomo, N. Kimura, T. Nojima, H. Aoki, Y. Iwasa, and M. Kawasaki, Nat. Mater. 7, 855 (2008).

${ }^{23}$ H. Shimotani, H. Asanuma, A. Tsukazaki, A. Ohtomo, M. Kawasaki, and Y. Iwasa, Appl. Phys. Lett. 91, 082106 (2007).

${ }^{24}$ J. T. Ye, S. Inoue, K. Kobayashi, Y. Kasahara, H. T. Yuan, H. Shimotani, and Y. Iwasa, Nat. Mater. 9, 125 (2010).

${ }^{25}$ K. Ueno, S. Nakamura, H. Shimotani, H. T. Yuan, N. Kimura, T. Nojima, H. Aoki, Y. Iwasa, and M. Kawasaki, Nat. Nanotech. 6, 408 (2011).

${ }^{26}$ L. Wang, M.-H. Yoon, G. Lu, Y. Yang, A. Facchetti, and T. J. Marks, Nat. Mater. 5, 893 (2006).

${ }^{27}$ H. Yuan, H. Shimotani, A. Tsukazaki, A. Ohtomo, M. Kawasaki, and Y. Iwasa, Adv. Funct. Mater. 19, 1046 (2009).

${ }^{28}$ Y. Yamada, K. Ueno, T. Fukumura, H. T. Yuan, H. Shimotani, Y. Iwasa, L. Gu, S. Tsukimoto, Y. Ikuhara, and M. Kawasaki, Science 332, 1065 (2011).

${ }^{29}$ G. Kresse and J. Furthmüller, Phys. Rev. B 54, 11169 (1996).

${ }^{30}$ G. Kresse and J. Furthmüller, Comput. Mater. Sci. 6, 15 (1996).
${ }^{31}$ J. P. Perdew, K. Burke, and M. Ernzerhof, Phys. Rev. Lett. 77, 3865 (1996).

${ }^{32}$ P. E. Blöchl, Phys. Rev. B 50, 17953 (1994).

${ }^{33}$ K. Ueno, H. Shimotani, Y. Iwasa, and M. Kawasaki, Appl. Phys. Lett. 96, 252107 (2010).

${ }^{34}$ See Supplemental Material at http://link.aps.org/supplemental/ 10.1103/PhysRevB.87.155151 for additional transport data.

${ }^{35}$ K. R. Kittilstved, W. K. Liu, and D. R. Gamelin, Nat. Mater. 5, 291 (2006).

${ }^{36}$ J. M. D. Coey, M. Venkatesan, and C. B. Fitzgerald, Nat. Mater. 4, 173 (2005).

${ }^{37}$ P. A. Lee and T. V. Ramakrishnan, Rev. Mod. Phys. 57, 287 (1985).

${ }^{38}$ P. Nozières, J. Low Temp. Phys. 17, 31 (1974).

${ }^{39}$ D. Goldhaber-Gordon, J. Göres, M. A. Kastner, H. Shtrikman, D. Mahalu, and U. Meirav, Phys. Rev. Lett. 81, 5225 (1998).

${ }^{40}$ T. A. Costi, A. C. Hewson, and V. Zlatic, J. Phys.: Condens. Matter 6, 2519 (1994).

${ }^{41}$ W. Felsch and K. Winzer, Solid State Commun. 13, 569 (1973).

${ }^{42}$ T. A. Costi, Phys. Rev. Lett. 85, 1504 (2000).

${ }^{43}$ M. Ungureanu, H. Schmidt, Q. Xu, H. Vonwenckstern, D. Spemann, H. Hochmuth, M. Lorenz, and M. Grundmann, Superlattices Microstruct. 42, 231 (2007).

${ }^{44}$ D. Wang, Q. Chen, G. Xing, J. Yi, S. R. Bakaul, J. Ding, J. Wang, and T. Wu, Nano Lett. 12, 3994 (2012).

${ }^{45}$ H. Suhl, Phys. Rev. 138, A515 (1965).

${ }^{46}$ V. Bhosle, A. Tiwari, and J. Narayan, J. Appl. Phys. 100, 033713 (2006).

${ }^{47}$ R. S. Thompson, D. Li, C. M. Witte, and J. G. Lu, Nano Lett. 9, 3991 (2009).

${ }^{48}$ See Supplemental Material at http://link.aps.org/supplemental/ 10.1103/PhysRevB.87.155151 for additional data on device performance. 